\title{
The Material World as an Aid for Proclus' Imagination
}

\author{
By Mark Stephenson
}

\section{Introduction}

In this paper, I seek to answer whether the physical world plays a role in Proclus's geometry, which is carefully situated and contextualized within his Neoplatonic ontological system. I argue that while geometry is formally independent from the sense world, the geometer is inspired by and utilizes things ontologically below true geometricals while making geometrical arguments, which is enabled by the structure of the Proclean system. This presents a novel approach that resolves the difference between Proclus' progressive view of the abstract nature of geometry and his use of geometry in practice in the sense world.

In Proclus's Neoplatonism, the epistemological and ontological status of mathematics relies heavily on an expansion of the analogy of the divided line. In this Platonic analogy, reality is divided into four ontological levels, ranging from the highest grades of reality that are exact and free of matter to the lowest grades that are inexact and enmattered; the highest realms occupy a greater portion of the line, while the lower are less significant. ${ }^{1}$ Each of these ontological realms is mirrored by an epistemological faculty. ${ }^{2}$ The higher two are the realms of intelligibles, further split between intelligibles, discerned by intellect, and understandables, by understanding; the lower two are the realms of perceptibles, split between perceptibles, sensed by belief, and likenesses, by conjecture. ${ }^{3}$ The distinction between these levels is most easily understood through an example: Consider a circle. In the realm of likeness exists the shadow or reflection of a circle; in the perceptible world exists a circle on a sheet of paper; in the understanding exists the set of all circles; and in the intellect exists the Platonic Form of circularity. Of the four realms, the upper three and their associated faculties are of particular interest to Proclus in the context of mathematics. Intellect, the highest realm, is that of the ideal Platonic Forms. ${ }^{4}$ The faculty that examines objects of the intellect is immediate and superior to all other forms of knowledge; likewise, the objects have an unmatched purity, for as Forms they are the essence of something's existence. Perception is the realm of

1 Plato, The Republic, trans. Benjamin Jowett (The Internet Classics Archive, Massachusetts Institute of Technology).

${ }^{2}$ Dmitri Nikulin, "Imagination and Mathematics in Proclus," Ancient Philosophy 28 (2008): 156-7. He notes that while the allegory of the line is native to Platonism, the introduction of the cognitive faculties is novel to Neoplatonism, formalized by Syrianus.

${ }^{3}$ Proclus Lycius, A Commentary on the First Book of Euclid's Elements, trans. Glenn R. Morrow (Princeton: Princeton University Press, 1992), 10-11.

${ }^{4}$ Which are supplied by the Nous, sometimes translated as the divine Intellect. 
material objects, which are inexact and divisible (i.e. not made of a single, pure being); the faculty of belief perceives these objects using the physical senses, "laying hold of the truth obscurely." 5 Proclus then posits that the objects of mathematics must be understandables, objects of the understanding, ${ }^{6}$ in the realm intermediate to intellect and perception:

Mathematical objects ( $\lambda$ ó $о$ o $)$... have an intermediate position. They go beyond the object of intellect in being divisible, but they surpass sensible things in being devoid of matter. They are inferior to the former in simplicity yet superior to the latter in precision. ... Nevertheless they are only images, imitating in their divided fashion the indivisible and in their multiform fashion the uniform patterns of being. ... Let this be our understanding, for the present, of the intermediate status of mathematical genera and species, as lying between absolutely indivisible realities and the divisible things that come to be in the world of matter. ${ }^{7}$

The faculty of understanding, which engages in mathematical thought, thus exhibits the same intermediacy as the mathematical objects: it is able to know the objects undiluted and as they truly are but does not reach their ideal essence. I believe that this focus on the intermediate nature of mathematicals welcomes the sort of analysis I will be engaging in, though more background is needed first.

Table 1: Proclus' ontological and epistemological hierarchies.

\begin{tabular}{|c|c|c|c|}
\hline Ontological Class & Faculty & Manifestation & Sciences \\
\hline 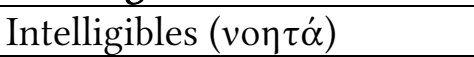 & 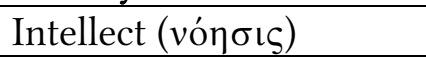 & Platonic ideal & Dialect \\
\hline 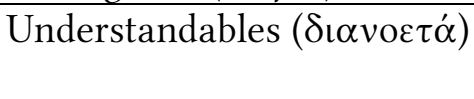 & Understanding $(\delta ı \alpha ́ \alpha \operatorname{col} \alpha)$ & Definition & $\begin{array}{l}\text { Arithmetic, } \\
\text { Geometry }\end{array}$ \\
\hline Imagined things & Imagination $(\varphi \alpha \nu \tau \alpha \sigma i \alpha)$ & Perfect instantiation & \\
\hline Perceptibles $(\alpha i \sigma \theta \eta \tau \dot{\alpha})$ & Belief $(\pi i \sigma \tau \imath \varsigma)$ & $\begin{array}{l}\text { Imperfect physical } \\
\text { instantiation }\end{array}$ & $\begin{array}{l}\text { Geodesy, } \\
\text { Physics, } \\
\text { Medicine }\end{array}$ \\
\hline 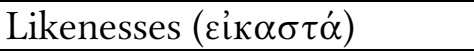 & Conjecture $(\varepsilon \imath \jmath \kappa \alpha \sigma i \alpha)$ & Image/reflection & \\
\hline
\end{tabular}

To clarify, while objects that are akin to each other may exist on different levels and have some level of connectedness, the form of the circle and the definition of the circle and the circle drawn on a sheet of paper are ontologically distinct objects. As this essay investigates the interpretation of these objects across ontological levels in both directions, I employ the following terminology: movement refers to any sort of cross-level interpretation of objects; instantiation refers to a downward motion from a general concept such as a Form or understandable into a specific instance; and abstraction refers to the upward direction.

An additional hierarchy that mirrors the epistemological hierarchy is that of the sciences. The axiomatic principles of each science are given by the science above it; for example, conclusions made using geometry inform the starting points of work in optics and geodesy. At the top of the hierarchy lies dialectic, "the study of being as being," which stands closer to "intellectual inspection"

\footnotetext{
${ }^{5}$ Proclus, Commentary, 3-4.

${ }^{6}$ Which are supplied by and part of the soul.

${ }^{7}$ Proclus, Commentary, 4-5.
} 
than any other science; "knowledge of these [immaterial] objects [i.e. the forms] is by far the prior science." ${ }^{8}$ Dialectic aims to reach non-discursive, unhypothetical understanding of the forms; this is in contrast with a science just below dialectic, general mathematics, which highly discursive and hypothetical by nature - that is, while mathematical study sets out axiomatic starting points (hypotheses) and (discursively) reaches new conclusions from the hypotheses alone, dialectic aims to know purely and unequivocally. ${ }^{9}$ To general mathematics (the science overarching all specific mathematical fields) below, dialectic supplies methods of mathematical inquiry ("analysis, division, definition, and demonstration"), allowing mathematical proof to be accepted as true. Proclus asserts - following Plato - that dialectic is the capstone of mathematics in the same way that intelligibles (the Forms) reign over understandables (such as mathematical objects). ${ }^{10}$ Gregory MacIsaac additionally points out that the concept of self-evidence, or how mathematicians recognize and decide upon their hypotheses, is a function of dialectic knowledge. General mathematics, following dialectic, supplies concepts such as ratio and equality to the two sciences below, arithmetic and geometry. ${ }^{11}$ Much has been written about the division of arithmetic and geometry in the Pythagorean and Neoplatonic traditions, but for brevity I will proceed directly to discussion of geometry as a science in relation to dialectic, since general mathematics mainly serves as a taxonomical convenience for comparing and contrasting arithmetic and geometry. ${ }^{12}$

To Proclus, the essential feature of mathematical inquiry, such as geometry, is discursive reasoning, which gives geometry infinite knowledge-generative power while simultaneously restricting the objects that can be directly studied by it and the variety of conclusions that it can achieve. He describes the discursive mode of mathematical thought as follows:

a life-giving activity, it unfolds and traverses the immaterial cosmos of ideas, now moving from first principles to conclusions, now proceeding in the opposite direction, now advancing from what it already knows to what it seeks to know, and again referring its results back to the principles that are prior in knowledge. ... The range of thinking extends from on high all the way down to conclusions in the sense world, where it touches on nature ... just as it rises from below and nearly joins intellect in apprehending primary principles. ${ }^{13}$

This passage emphasizes the nature of discursive thought: It is bidirectional, as it works both towards generality and specificity across the entire range of the ontological level of understandables; and it is self-contained, building upon a set of hypotheses in a self-contained system of thought. It is possible and even commonplace to reach the boundaries where the understanding meets the intellect and the sense world, but those boundaries can never be crossed as doing so would require the introduction of hypotheses not from mathematics, which violates the

${ }^{8}$ Proclus, Commentary, 9-10.

9 D. Gregory MacIsaac, "Geometrical First Principles in Proclus' Commentary on the First Book of Euclid's Elements," Phronesis 59, (2014): 91-92, 96.

${ }^{10}$ Proclus, Commentary, 42.

${ }^{11}$ Proclus, Commentary, 60-61.

12 Luc Brisson, "Chapter 18 of the De Communi Mathematica Scientia Translation and Commentary," in Iamblichus and the Foundations of Late Platonism, ed. Eugene Afonasin, John Dillon, and John F. Finamore (Leiden: Brill, 2012), 37-50; Proclus, Commentary, 57-61.

${ }^{13}$ Proclus, Commentary, 18-20. 
nature of discursive thought and thus mathematics as a whole; by nature, the result of discursive thought must be completely traceable to the starting hypotheses alone, and in crossing ontological boundaries new hypotheses must be added to give an object new properties when moving it into a different realm. As such, the objects upon which mathematics acts are equally limited to objects of understanding given the parallel nature of ontological and epistemological structures. Proclus follows the same argument for the ontological status of geometricals as for mathematicals, placing them as understandables with properties that are a mixture of those of intelligible forms and objects of the sense world. ${ }^{14}$ To be clear, these true geometrical objects are essentially definitions with the properties that follow from the definition: the circle is not the image of a circle but the property of all points being equidistant from a center point and all the properties that follow from that. I believe that this view of geometry is very progressive; the emancipation of objects from a particular representation is responsible for many discoveries of modern mathematics. However, this begs the question: "What is the ontological status of the envisioned, particular geometric form?" It has neither the inexact materiality of a physical object nor the generality of an understandable.

To answer this, Proclus relies heavily on an important Neoplatonic addition to the ontological hierarchy: the imagination $(\varphi \alpha \nu \tau \alpha \sigma i \alpha)$, the plane upon which one can envision and manipulate perfect instances of geometrical objects ( $\lambda$ ó "projects" the understandable mathematicals upon the plane of imagination. If the understanding is the intermediary between intelligible forms and sense objects, "imagination, then, is intermediate between sense perception and discursive reason: with the former, imagination shares the capacity to represent geometrical figures as extended; with the latter, it shares the capacity to represent its object as unchangeable according to its properties," as described by Nikulin. ${ }^{16}$ Setting the imagination within the hierarchy of manifestations of the circle: In the intellect exists the Form of circularity; in the understanding, the definition of a circle; in the imagination a perfect but manipulatable image of a circle; and in the sense world, an imperfect drawing of a circle. As Nikulin addresses, the projection of objects to the imagination from the understanding unveils hidden properties but does not capture the entirety of the object as it exists in the understanding, though through discursive thrught it can aid in more completely accessing the properties of an object in the understanding. This productive quality of the imagination is a novel advancement from the Aristotelian imagination that only served to abstract from the sense world. ${ }^{17}$ The connection between the imagination and understanding in the Proclean paradigm is very important to mathematical epistemology but does not grant the imaginary image the status of a true mathematical as it is not an understandable proper, as I intend to argue throughout this essay. To be clear: Proclus classifies true geometrical study as a function of the understanding and understanding alone. When comparing the circle across ontological levels, he writes,

\footnotetext{
14 Some scholars theorize that the first prologue was originally written as an introduction for another mathematicians text, explaining the great degree of overlap between the two prologues, with the second being more geometrically focused.

15 Proclus, Commentary, 13.

${ }^{16}$ Nikulin, "Imagination," 160.

${ }^{17}$ Nikulin, "Imagination,” 164-6.
} 
the circle in the understanding is one and simple and unextended, and magnitude itself is without magnitude there, and figure without shape; for such objects in the understanding are devoid of matter. But the circle in the imagination is divisible, formed, extended ... whereas the circle in sensible things is inferior in precision, infected with straightness, and falls short of the purity of immaterial circles. ${ }^{18}$

In this way, he clearly distinguishes the projections in the imagination as distinct from the true mathematical understandables.

Previous scholarship has highlighted Proclus' use of the imagination as unique ${ }^{19}$ in that the figures it envisions are instantiations of geometric forms of the understanding, rather than abstractions from the physical as in the Aristotelean conception; these figures may then be manipulated in the imagination to produce knowledge of the understandables. Orna Harari says that this "account, in which mathematical objects are projections onto the imagination of forms that pre-exist in discursive reason, secures the status of mathematics as an explanatory science, hence as a science in the strict sense," thus the relationship between the imagination and understanding is key to the entire philosophical structure that Proclus builds around mathematics. ${ }^{20}$ Guy Claessens argues that Proclus' conception of imagination as a productive aid as opposed to a sensory receptacle informed Kepler's Harmonices Mundi, a view contrary to the orthodoxy of early modern mathematical philosophy. ${ }^{21}$ And Nikulin likewise champions the significance of the imagination working in support of discursive reason in the understanding. While I agree that Proclus's conception of the imagination is a productive augmentation subordinate to the understanding, in what follows I will pursue an analytical path more similar to David Rabouin: I find that geometric practice is informed by the geometer's experience with the sense world to a greater degree than the core tenets of Proclus' philosophy often let on, and I apply this idea to the imagination. ${ }^{22}$ I find that the imagination still appears as a receptacle for sense objects similar to the Aristotelian practice, though in a way that preserves the well-established relationship between imagination and understanding in Proclus' ontological schema. Through this, I expand upon our understanding of the utility of Proclean mathematics.

More broadly, I use this as a means to address why Proclus wrote a commentary on the geometrically-focused Elements from an ultimately dialectical perspective, despite MacIsaac rightfully pointing out that being a dialectician or even familiar with dialectic is not necessary to do mathematics in the Proclean framework. ${ }^{23}$ Proclus himself says that the geometer, like the

18 Proclus, Commentary, 54-5.

${ }^{19}$ Anne Sheppard, "Phantasia and Mathematical Projections in Iamblichus," Syllecta Classica, Vol. 8 (1997): 113120. Or at the most explicitly formulated conception, formalizing ideas present in near-contemporaries such as Syrianus and Iamblichus.

${ }^{20}$ Orna Harari, "Proclus' Account of Explanatory Demonstrations in Mathematics and its Context," Archiv $f$. Gesch. d. Philosophie 90 (2008): 163.

${ }^{21}$ Guy Claessens, "Imagination as Self-knowledge: Kepler on Proclus' Commentary on the First Book of Euclid's Elements," Early Science and Medicine 16 (2011): 179-99.

22 David Rabouin, "Proclus' Conception of Geometric Space and Its Actuality" in Mathematizing Space, ed. Vincenzo De Risi (Switzerland: Springer, 2015), 105.

${ }^{23}$ MacIsaac, "First Principles," 47; Proclus, Commentary, 93-4. 
physician and physicist, need only concern themselves with the first principles of their studies and the ontological realms they work in. ${ }^{24}$ Despite this, Proclus' entire text appears to be a dialectical, multileveled take not just on geometry proper, but how geometry is situated ontologically and approached epistemologically. This meta-geometrical nature may seem unusual or unmathematical to anybody familiar with modern math textbooks derived from Euclid's archetype, but this is because Proclus is commenting on what he sees as the two key aspects of Euclid's Elements: an "economically and orderly arranged" guide to geometry, and a guide to methods of mathematical argument. The former leaves less room for commentary, by Proclus' own admission: "If you add or take away any detail whatever, are you not inadvertently leaving the way of science and being led down the opposite path of error and ignorance?" 25 Indeed, Proclus' more mathematical commentary on the proofs is largely composed of analysis of others' alternate proofs and if they are satisfactorily geometrical. Thus, the latter (Euclid's implicit demonstration of methodologies) welcomes commentary outside the geometrical for it is explicitly described by Proclus dialectical study; the far reaching prologues certainly aim to situate geometry ontologically, and even the later commentary on proofs and their methods is an epistemological survey, not a novel geometrical work. If one wants a text that builds upon the strictly geometric aspect of the Elements, Proclus suggest Archimedes' Sphere and Cylinder and Apollonius' Conics; but this is not the sort of text Proclus set out to write. ${ }^{26}$

I set out to show that while geometry as a discursive system has no reliance on anything outside of the understanding, geometric thought, as Proclus describes it, not only requires imagination as a tool for reflection of the understanding but also as a faculty that interprets and utilizes the sense world. This follows from Proclus' situation of geometry within the larger ontological structure accessed by dialect, as opposed to limiting analysis to the space between the imagination and understanding that most modern scholarship has focused on. In doing so, this grants validity to a wide range of geometer's actions, few of which strictly operate in the range of geometry proper, the understanding.

With the necessary background in Proclean ontology already given, I will first (II. Ontologically Flexible Geometricals) show that geometrical objects are defined in a way that implicitly enables movement across and within ontological realms; I then argue that due to the ontologicalepistemological parallelism in Neoplatonic philosophy, there must be some epistemological way of engaging with these flexible objects. Next (III. The Geometer's Limitations) I show that Proclus accepts that geometers often engage in geometrical study outside of the realm of geometry proper in the imagination but are still able to produce geometrical knowledge. Finally (IV. Geometrical Reliance on the Sense World), I propose that this may be extended into the sense world; I utilize examples from definitions, diagrams, and the lower sciences to argue that not only is it possible to engage in geometry in the sense world, but it is commonplace.

\footnotetext{
24 Proclus, Commentary, 92-3.

25 Proclus, Commentary, 69-70.

${ }^{26}$ Proclus, Commentary, 71.
} 


\section{Ontologically Flexible Geometricals}

I begin by showing that Proclus defines geometrical objects in a way that enables movement between ontological realms. Depending on the realm a geometrical object is in, the properties of an object and its relationship to other geometrical objects change accordingly; no object is excluded from cross-realm movement. Once shown, I argue that his epistemology is structured to mirror this ontological flexibility.

Proclus' description of geometrical objects includes commentary on their nature outside the strictly geometrical understanding and even relates objects across levels of the ontological spectrum. This is primarily seen in enmattered forms, ${ }^{27}$ a class that includes any instantiation of abstract mathematicals; both sense objects existing in physical matter generated by nature and imaginary objects projected onto the material plane of the imagination are included in this class. Unlike those ideals existing in the realm of the soul which are perfect and unified, these material forms are "dispersed among their several subjects" and "possess only an imported unity"; that is, instantiation of a mathematical abstract always occurs with the simultaneous instantiation of other abstracts not necessarily from the mathematical discipline (e.g. a circular dirt mound in the sense world has geometric properties of circles - though imperfect - and agricultural properties of soil and physical properties of matter, among many others) ${ }^{28}$ Proclus gives the example of a solid body and bounding planes: The solid body exists as body-ness in the understanding and the planes likewise exist as plane-ness; the relationship of bounding can be conceived of but does not alter the fundamentally independent ideas of body- and plane-ness. However, "in our thought or perception," the bounding planes become an inseparable part of the imagined or sensed solid body; the unbounded body cannot be perceived and thus the bounding surfaces are subsequent to and a product of the instantiated, finite body.

This example leads to a larger classification of geometricals along the continuum between the intelligible and sensible in Proclus' ontology. In general, he defines the continuum as a scale between the Limit and the Unlimited (which tend toward intelligibility and sensibility respectively), in which the point is the limit of the line, the line the limit of the plane, and the plane the limit of the solid body. ${ }^{29}$ The Limit is identified with singularity, primacy, and simplicity; in the immaterial realm of mathematics,

the limiting factors have an essential priority over the things that are limited; as being less divisible, more uniform, and more sovereign; for among immaterial forms unity is more perfect than plurality ... and what bounds more perfect than what gets its limit from something other than itself. ${ }^{30}$

As such, the point is the most Limit-like of the elements; however, this status is not absolute: Objects simply "sit close toward the Limit, others incline towards the unlimited," but they all take part in

\footnotetext{
${ }^{27}$ Forms as various geometric objects, not to be confused with Platonic capital-F Forms.

${ }^{28}$ Proclus, Commentary, 86.

${ }^{29}$ Solid body as a translation of $\sigma \omega \dot{\mu} \alpha$, a three-dimensional object; Morrow translates it as body.

${ }^{30}$ Proclus, Commentary, 86.
} 
both to some extent even within a single ontological realm. ${ }^{31}$ Thus even the completely partless point when considered solely in the "higher realities" (i.e. the intelligible realm) has some aspect of the Unlimited with a generative power; that is, a point may be found anywhere within a continuous object bounded by points. On the other end of the spectrum (still considering only the higher realities), the solid body inclines more towards the Unlimited as it is highly divisible and dimensional. The line and plane are intermediate to the point and solid body and as such have more equally mixed amounts of the Limit and Unlimited. ${ }^{32}$ As the ontological scale is descended to the material of the imagination or sense perception, those objects that take part more strongly in the Unlimited, like the solid body, are minimally affected because their properties are better expressed in material instantiation. For more Limit-like objects such as the point, only "traces of the most exalted principles" such as exactness and indivisibility are preserved, though Unlimited qualities like the point's continuous presence in lines, plane, and bodies are amplified. For example, a point may be found at any coordinate within the limits of a material body, at any location on the surface, or any position along the edges. ${ }^{33}$ Recalling the example of the solid body and the plane, the planes on the outer limits of the body are but a few of an infinite number of planes that can be found in the body, just as the points at the corners are but a few of the infinite points within the body. Thus in matter, the limits lose their status as independent and primary objects; instead they emerge from the more composite objects that remain better preserved in matter.

Proclus makes clear that despite some people's claims to the contrary the limits do still exist in the material realm. If one is not convinced that a line exists on the edge of an imperfect material cube, they may be more willing to accept the axis about which a celestial body orbits as an exact line in the material world. ${ }^{34}$ But Proclus takes this a step further and argues that all forms, limiting or limited, are real and can be moved across all ontological levels:

Most people, observing that limits exist imperfectly in limited things, have a confused conception of their being. [The Stoics] say that they are only abstracted by reflection from sensible things, others that they have no existence apart from our thoughts. But the forms of all of them do exist in the intelligible world, they exist in the orders of the soul, they exist in nature and, last of all, in bodies. ${ }^{35}$

I believe that an important aspect of Proclus' ontology emerges from this insistence on the presence of the forms of limits in the perceptible world, combined with the Limit-Unlimited dual nature of all geometrical objects even within a single ontological rung: Proclus considers these objects to be existent across geometrical and non-geometrical ontological realms and malleable within them, as opposed to only existing within and across realms due to an observer's interpretation. The processes of movement across realms and malleability within them are not discrete; Proclus describes the

\footnotetext{
${ }^{31}$ Proclus, Commentary, 87.

32 Proclus, Commentary, 88.

${ }^{33}$ Proclus, Commentary, 89.

${ }^{34}$ Proclus, Commentary, 89-90.

35 Proclus, Commentary, 91
} 
Limited/Unlimitedness of the point as fluid and context-based across and within ontological planes, not a binary function between the material and immaterial.

Proclus suggests and actively demonstrates that the study and interpretation of the forms across the epistemological hierarchy is has a fluidity that matches the forms' ontological flexibility. While the discursivity of mathematics restricts mathematical argument to objects of mathematics which are solely of the understanding, the borders of mathematics are acknowledged by Proclus ${ }^{36}$ and often exceeded, as seen by his discussions of the manifestation of mathematicals in the sense world. MacIsaac expands on this idea in his study of Proclus' use of boundaries (horos), a word used to describe both the geometrical boundaries of a figure and the boundaries between sciences; in the latter, epistemological definition, the result of one study crosses over a horos as the predicate for a subordinate study or science. ${ }^{37} \mathrm{He}$ indicates that there is a faculty that works to move between manifestation of forms in material and imaginary contexts; this augments the responsibilities of the dianoetic faculty at the interface between the plane of imagination and the understanding, which is more commonly focused on in modern scholarship. I think that modern scholarship's focus on the understanding/imagination interface comes from a section Proclus writes about the source of the forms seen in the imagination:

We must therefore posit the soul as the generatrix of mathematical forms and ideas. And if we say that the soul produces them by having their patterns in her own essence and that these offspring are the projections of forms previously existing in her, we shall be in agreement with Plato and shall have found the truth with regard to mathematical being. ${ }^{38}$

If this were not the case and the essence of mathematical forms and truth resided in the sense world, Proclus argues, they would be too foreign, unstable, and inexact to yield the precision natural to mathematics; obviously, the source of the forms must match the Platonic top-down ontological hierarchy. However, I see nothing in the prior passage and its context that prevents the ability to encounter and interpret between sense objects and those of the imagination; Proclus simply says that abstraction from the sense world is not the ontological source of those things in the imagination. Harari explains the ontological hierarchy as a causal relationship ("in viewing mathematical objects as projections of pre-existent forms, Proclus in fact conceives of them as effects of productive causes") that extends into physical instantiations. ${ }^{39}$ This is reflected in Proclus' discussion on the ontology of the point: "As it descends on the scale of being ... the point takes on the character of divisibles"; similarly, "For all ideas [of the intellect and understanding] when they flow into matter ... forsake their native simplicity." 40 Thus the point in the material world has a direct ontological lineage leading to the ideal point; this is regardless of whether the observer senses the point as her first interaction with it. A faculty that simply detects the relationship between the

\footnotetext{
${ }^{36}$ Recall that math "extends from on high all the way down to conclusions in the sense world, where it touches on nature ... just as it rises from below and nearly joins intellect in apprehending primary principles." Proclus, Commentary, 19.

${ }^{37}$ MacIsaac, "First Principles," 58-67.

${ }^{38}$ Proclus, Commentary, 13.

${ }^{39}$ Harari, "Demonstrations," 155-7.

${ }^{40}$ Proclus, Commentary, 92-3.
} 
sensible point and that which it is borne from without rewriting the ontological structure is compatible with and evident in his philosophy, and I believe that Proclus utilizes it throughout his discussion of geometric practice. Dmitri Nikulin tells us "cognitive faculties are defined by their objects, and imagination is determined in mathematics by geometrical figures, which have ... features of both 'passive' physical bodies and of thinkable logoi." ${ }^{41}$ Applying this relationship to ontologically flexible objects implies the existence of a faculty to handle them.

\section{The Geometer's Limitations}

I proceed by showing that Proclus does not expect geometers to access geometricals directly, but still considers them capable of generating geometrical knowledge via a lower plane, the imagination. This is established in preparation for showing the extension of this idea into the physical world, where indirect interaction with geometry acts both as a source of inspiration for geometrical activity and as a realm where geometrical knowledge can be produced.

Proclus recognizes that a key property of good mathematical judgment is the ability to generalize as much as possible while maintaining the necessary degree of specificity. For example, while it is not false to claim that "the isosceles triangle has the sum of its angles equal to two right angles," the claim demonstrates a poor grasp of the subject matter since all triangles - not just isosceles - have this property. ${ }^{42}$ Likewise, Proclus argues that a single demonstration should not be too broad, with the example that one may not be made that applies to figures and numbers due to their different underlying genera; this is due to the separation of arithmetic and geometry in Neoplatonism. ${ }^{43} \mathrm{He}$ observes that there is an incongruence between the work of an ideal geometer and what geometers produce in practice. The dialectical draw toward the universal in individual demonstrations is mirrored by a draw towards universals in the form of understandables in general; premises and demonstrations should be of the same nature, so when attempting to demonstrate properties of understandables, the starting points must be as universal as possible. Thus, the "perfect culmination of geometric inquiry":

If [the understanding] should ever be able to roll up its extensions and figures and view their plurality as a unity without figure, then in turning back onto itself it would obtain a superior vision of the partless, unextended, and essential geometrical ideas that constitute its equipment. $^{44}$

This "gift from Hermes" would emancipate the geometer from the use of the imagination but implies that the ultimate universality demanded of the geometer is not reachable. This also offers a

${ }^{41}$ Nikulin, "Imagination," 164.

42 Proclus, Commentary, 14.

${ }^{43}$ Proclus, Commentary, 33. The separation of arithmetic and geometry is less clear cut than presented here due to Proclus' acknowledgement elsewhere that the two halves of mathematics commonly inherit some principles from general mathematics. Potentially, the Proclean resolution is that such demonstrations must be made in the realm of general mathematics, but given that symmetries and equivalences (those principles received from general mathematics) are - as far as I can tell - not considered to be objects of Neoplatonic mathematics in the same way that units and points are, it is unclear with what objects such a demonstration would actually be made.

${ }^{44}$ Proclus, Commentary, 55. 
clue as to why Proclus uses dialectic so extensively: He is making clear the process aided by imagination that occurs when attempting to reach pure geometric study. That process is necessary in the "true geometer's ... goal to arouse himself to move from imagination to pure and unalloyed understanding," so it is expected that a text not just about geometry but how to engage with geometry such content would be included. As MacIsaac points out, Proclus gives us "a description of how the science of dialectic understands the truths known by geometry and how these truths belong in a fuller sense within dialectic." ${ }^{45}$

While the ideal geometer would directly study geometric objects of the understanding, Proclus adds imagination to the Platonic epistemological hierarchy in acknowledgment of the shortcomings of the human mind. However, the imagination is not purely an intermediary stepping-stone to reach direct thought in the understanding and geometric knowledge; Proclus allows it to be an endpoint that produces geometrical knowledge without explicitly returning to the understanding:

When, therefore, geometry says something about the circle ... let us not say that it is instructing us either about circles in the sense world, for it attempts to abstract from them, or about the form in the understanding. For the circle [in the understanding] is one, yet geometry speaks of many circles, setting them forth individually and studying the identical features of all of them; and that circle [in the understanding] is indivisible, yet the circle in geometry is divisible. Nevertheless we must grant the geometer that he is investigating the universal, only this universal is obviously the universal present in the imagined circles. Thus while he sees one circle [in the imagination], he is studying another, the circle in the understanding, yet he makes his demonstrations about the former. ${ }^{46}$

Although this passage is somewhat ambiguous on whether geometry studies universals or images in the imagination, I continue to agree with Morrow's reading which finds that geometry certainly studies universals, residing in the understanding; Proclus is explicit that "mathematical objects are ... understandables." 47 To resolve this, I read "geometry" in the quoted passage on circles as a reference to the manipulation of geometricals in the imagination, not as nature of the essential elements of the science of geometry proper; this is consistent with his repeated designation of understandables as indivisible and forms of the imagination as divisible. Proclus grants that arguments using instances of the universals (the universals being understandables that are true mathematical objects) generate knowledge about the universals. A convenient byproduct of this structure is that this broadly enables people to make geometric arguments, which would be otherwise nearly impossible in a framework that requires arguments to be made in the understanding exclusively. ${ }^{48}$ Harari approaches this issue from a different angle, proposing that geometry "should study both reason-principle that pre-exists in discursive reason, and its immanent manifestation in the imagination as discursive reason cannot apprehend the forms it contains

${ }^{45}$ MacIsaac, "First Principles," 93

${ }^{46}$ Proclus, Commentary, 54.

${ }^{47}$ Proclus, Commentary, 11.

${ }^{48}$ While perhaps Proclus realized that this must be a byproduct of his epistemological system, arguing that it is the reasoning for the system's structure would be highly un-Platonic as the examination of reality shouldn't dictate the nature of reality. 
without the aid of the imagination." 49 While this interpretation is semantically different in its definition of geometry, the general conclusion is the same: "the relationship between the substrate and its attributes is no longer accidental; it is the necessary relation between a cause that produces in virtue of its being and its effects." Nikulin agrees: "Imagination thus facilitates discursive reason's study of a mathematical object, which ... is unmoved, not produced, and indivisible, but which has to be 'alienated' from itself, set into (imaginary) motion, brought into an (imaginable) extension, and placed in (geometrical) matter." ${ }^{50}$ Thus, there is no limitation that geometrically productive thought must take place or even end in the realm of geometry, as the imagination does not study understandables but still produces knowledge about them.

Proclus highlights the results of imaginative thought as being able to abstract directly into the understanding, but this precedent can be applied at other interfaces (i.e. between the realms of sense and imagination), transitively implying a route for arguments from the material to understanding. The universal being represented by instances of imaginary objects is in the understanding thus allowing for abstraction into the understanding, while the universal represented by instances of sense objects is in Nature, outside of the epistemological scale and thus not directly abstractable into geometry proper due to the different substrate material. The movement from "the lowest forms of knowledge" - sense perception - to "the central position in the scale of knowing" - imagination - is also an "attempt to abstract," but an abstraction from instantiation in the material of the sense world to instantiation on the plane of imagination, as opposed to an abstraction from instances of the imagination to a universal. ${ }^{51}$ Both in the sense world and imagination instances are manipulated, differing only in that the former instance is made of imperfect, material matter while the latter instance is a perfect, immaterial substance. This suggests that - as with the abstraction from the imagination to understanding - the abstraction from the material to the imagination only needs to be possible, not actively realized, for geometric argument to occur.

\section{Geometrical Reliance on the Sense World}

While I have shown that geometrical objects can be interpreted across ontological levels via the imagination, I take this a step further by arguing that movement as far down as into the sensible world is commonplace and even necessary in geometrical argument and study, as evidenced by Proclus' frequent citation of sense-based arguments. Proclus has clearly established a willingness to accept geometer's arguments made in the not-strictly-geometrical realm of imagination as geometrical, acknowledging that the direct manipulation of understandables is only accessible to the god-geometer. But these demonstrations are not only allowed but expected to be even further from pure mathematics:

Since mathematics occupies a middle position between the intelligible and the sense worlds and exhibits within itself many likenesses of divine things and also many paradigms of physical relationships, we must observe the threefold character of its demonstrations, some being more

\footnotetext{
${ }^{49}$ Harari, "Demonstrations," 160-2.

${ }^{50}$ Nikulin, "Imagination," 145.

51 Proclus, Commentary, 52
} 
intuitive, some more discursive, and some approaching the nature of opinion (i.e. sense perception). ${ }^{52}$

This varying level of precision in the arguments, as with the geometrical objects themselves, must be interpretable back into true mathematical understanding, which does not permit persuasion as reasoning; this implies that mathematical truth is present in the sense world which allows the role of a good mathematician to be read not purely as one who strives to reach understanding, but one who elucidates the pathway to the understanding from the medium of the argument's hypotheses. This aligns with Proclus' view on the extensiveness of geometry specifically, that "it is coextensive with all things, [and] applies its reasoning to all," and while "the forms of the understanding constitute its essence ... through the middle region it ranges upwards and downwards to everything that is or comes to be." 53 While he occasionally indicates that "everything" is as far-reaching as political philosophy and morality, I choose to focus on the portion of the "middle region" that is actively addressed through examples of the multicharactered, geometrically-reaching demonstrations.

Several passages indicate material inspiration as a starting point for geometric argument. Mathematical thought is characterized as "beginning with reminders from the outside world" and "awakened to activity by lower realities," then traversing up and down ontological levels in a dianoetic manner; it is unique from other forms of thought in that it always "refers its results back to the principles that are prior in knowledge," i.e. those starting points inspired by the sense world. ${ }^{54}$ Proclus later reiterates: "Although we are stirred to activity by sense objects, we project the ideas within us" to reveal forms on the levels of the understanding and divine intellect; he follows with a redescription of the imagination as the reflection of objects already present in the soul. ${ }^{55}$ The key takeaway I strive to highlight here is that physical inspiration is neither incompatible with nor in conflict with the soul as the source of imaginary figures; rather, the sense world is freely included in Proclus' epistemology of geometry. ${ }^{56}$

Proclus compares many definitions and constructions of the line, as it is the simplest geometrical figure that may be defined both in terms of what it is and isn't made of (points and breadth, respectively); through these, he demonstrates the production of mathematical knowledge from different ontological levels as well as the mathematical consequences of alternative starting points. The most basic definition, given by Euclid, acts in the domain of understanding by establishing equivalence between the line and its properties: "A line is length without breadth." ${ }^{2}$ While Proclus spends a paragraph dissecting the definition, he immediately follows with additional, more concrete definitions; at a minimum, this implies that Proclus finds it necessary to acknowledge how others have conceptualized the line and perhaps presents it for the reader's understanding. He accepts a "magnitude extended in one direction" as perfectly descriptive, even though it is an active

\footnotetext{
52 Proclus, Commentary, 35.

${ }^{53}$ Proclus, Commentary, 62-3.

54 Proclus, Commentary, 18-9.

${ }^{55}$ Proclus, Commentary, 140-2.

56 Glenn R. Morrow, "Introduction” in A Commentary on the First Book of Euclid's Elements, lxii-lxiii.

${ }^{57}$ Proclus, Commentary, 96-7
} 
process of the type often associated with operations of the imagination; however, he relegates "the flowing of a point" as a definition of the material line only, owing to the fact that it is constructed as continuous collections of points, as all material objects are to allow for arbitrary divisibility. The former, residing in the imagination, is easily labeled "perfect" because of the generally unambiguous abstraction from imagination to the understanding. In the case of the latter, Proclus does not dismiss this imperfect definition as an unmathematical statement, as this mathematical conception of the material is on the continuum of objects adaptable into true geometricals and within the types of arguments pertaining to geometry may be made; however, it is imperfect because it is farther removed from the understanding than necessary.

A similar precedent for more physically-focused definitions arises when Proclus' considers Plato's definition of the straight line: "that whose middle intercepts the view ${ }^{58}$ of the extremes." ${ }^{9}$ Without criticism or acknowledgment of its use of the senses, Proclus unequivocally illustrates the definition by interpreting "view" as the physical act of seeing done by eyes. ${ }^{60} \mathrm{He}$ expands upon this by claiming "this property ... affords a proof that in the realm of being ... the middle orders of things" have a connection to the material similar to their connection to the most abstract. This demonstrates two key features: mathematical knowledge and proof from the sense world - well outside the strictly mathematical - and the ability of mathematical forms to be moved in both directions across the ontological spectrum.

Despite material inspiration generally being departed from as an argument unfolds, the sense world may continue to play a significant role further into geometric practice; this is primarily seen in the use of diagrams. Diagrams are interesting in the context of Neoplatonic geometry, as they are physical and particular, yet they lead to abstract and generalized conclusions. ${ }^{61}$ They hold a unique epistemological status as well. Unlike other physical sources of inspiration that may give the fledgling geometer a loose conception of a circle or parallelism which is then formalized, the diagram is explicitly provided in the sense world to aid imagination with more complex constructions; it offers a framework for imagining the perfect and precise construction. While diagrams in Proclus are never presented without an accompanying textual construction, this does not make them are superfluous; they are included because Proclus considers them necessary sensible aids, enabled by his willingness to grant geometricy to arguments made in realms well below geometry proper. As Manders notes, from ancient times to the $19^{\text {th }}$ century arguing directly from geometric diagrams was considered mathematically valid; even if Proclus offered more rigor to his constructions, he was not operating in a truly axiomatic manner since conclusions are reached with some reliance on a basic level of correctness in his diagrams, regardless of accompanying textual description. ${ }^{62}$

58 "View" is $\dot{\varepsilon} \pi \imath \pi \rho \sigma \theta \varepsilon \tilde{\varepsilon}$, translatable as astronomical occultation or a more general sense of intercepting the view.

${ }^{59}$ Proclus, Commentary, 109-10. Note that helixes and other curves are included in Euclid's broad definition of lines, contrary to the typical modern definition.

${ }^{60}$ Perhaps unsurprising, given Neoplatonists' unwillingness to oppose their understanding of Plato's teachings.

${ }^{61}$ Dwayne Raymond, "From a Particular Diagram to a Universal Result: Euclid's Elements, Book 1," Apeiron, vol. 44 (2001): 211-218.

${ }^{62}$ Kenneth Manders, "The Euclidean Diagram (1995)," in The Philosophy of Mathematical Practice, ed. Paolo Mancosu (Oxford University Press, 2008), 87-8. 
In simple cases, diagrams are pedagogic tools: Certainly, the god-geometer would not find them useful, but for the new geometer sacrificing generality and precision is acceptable to further develop geometric self-evidence and intuition. In more complex cases, diagrams also provide a framework for the imagination to conceptualize difficult constructions. Rabouin presents a number of examples of impossible diagrams (e.g. those depicting infinite lines or intersecting parallel lines) in Proclus and Euclid, noting that additional levels of movement into the imagination are needed: "It is therefore legitimate to distinguish the figure, in the sense mentioned above, from the diagrammatic configuration presented to our imagination in order to conduct the proof." ${ }^{3}$ Nikulin addresses the infinite in the same way: while the drawn line cannot be extended forever, the imagination can read the supposedly infinite line as actually infinite. ${ }^{64}$ While more advanced, these diagrams are still a stepping stone to aid the geometer in the pursuit of conceptualization in higher ontological strata. Proclus supports this view when claiming that the geometer should strive to work in purely in the realm of understanding: after conceding that the imagination is necessary to conceptualize understandables, he follows that we go a step lower to diagrams and illustrations to aid imagination as part of the same extension and unwrapping of the understanding.

Implicit ontological and epistemological access of geometry from the sense world occurs through the study and practice of sciences below geometry. In the same way that geometry inherits from general mathematics and Dialectic, lower sciences inherit their first principles from geometry in Neoplatonic epistemology. In what may be called applied geometry - subjects such as mechanics and optics - geometry "is bound up with sensible things and operative in them"; they are inferior due to the material inexactness introduced to the principles furnished by the more exact science of geometry. ${ }^{65}$ I propose that the same implicit ontological abstraction occurs when leveraging geometry in manipulations of a lower science as when interpreting arguments made in the imagination and below as a source of geometric truth. ${ }^{66}$ This is not a far-reaching claim: Proclus' passages ${ }^{67}$ that establish geometrical thought and proof as sometimes bordering on the inexactness of the material are often followed by description of the sciences just over the generally blurry border. Furthermore, the subordinate sciences are unquestionably considered fields of mathematics, despite not being studies of mathematical understandables: Euclid's works on optics, catoptrics, and music are all labeled as such, as are the perceptible fields of math in Geminus' classification. ${ }^{68}$

Proclus' description of the practice of these lower sciences is presented as a geometrical and truth-generating activity:

${ }^{63}$ Rabouin, "Geometric Space,” 119-22.

${ }^{64}$ Nikulin, "Imagination,” 168-9

${ }^{65}$ Proclus, Commentary, 19-20, 59.

${ }^{66}$ Nikulin, "Imagination," 158-9 suggests that this is only possible in geometry and its inferiors, and not those science borne of arithmetic: "Both arguments that account for the separation and distinctness of arithmetic and geometry as disciplines refer to the difference in the presence and appearance of otherness as materiality and infinity in numbers and figures. In particular, unlike numbers, geometrical figures are infinitely divisible and associated with a kind of matter, which makes them extended and perceptible as visualizable and extended.”

${ }^{67}$ Proclus, Commentary, 20, 35, 62.

${ }^{68}$ Proclus, Commentary, 69, 38. 
When it touches on the material world it delivers out of itself a variety of sciences - such as geodesy, mechanics, and optics - by which it benefits the life of mortals. Through these sciences it has devised instruments of war and defense for our cities, ... taught how to measure distances by land or sea, constructed balances and scales for determining arithmetical equality when a city needs it ... and many things incredible to men it has unveiled and made credible to all. Recall what Hieron of Syracuse is said to have said about Archimedes [upon his designing of a method to move a heavy ship]: "From this day forth we must believe everything that Archimedes says." ... Many of our predecessors have recorded such things in praise of mathematics, and for this reason we have presented here only a few of the many facts we might have cited to show the range and utility of geometrical knowledge. ${ }^{69}$

A key feature of this passage is that outcomes are expressed as geometry being applied through a lower science as opposed to results of a lower science that has acquired its first principles from geometry; this is a clear instance of the discursivity of geometric thought - that has already been shown to move through the imagination - moving into the sense world and back to geometry proper. The apocryphal story about the ship and others about applied geometry all conclude in the same way: acceptance of the outcomes of geometry after some proof of its utility made in the sense world. The outcomes are not posed as instances of empirical evidence for geometry; rather, they are shown as proof of the knowledge-producing nature of geometry ("we must believe everything"). While an apocryphal quote by a non-geometer and other references to these things being understood by laypeople ("made credible to all") seems to weaken claims of the geometric value of the passage, I argue the opposite: We already know that the mathematician can produce mathematical knowledge without a godlike grasp of the understanding, so instances of nonmathematicians accessing geometrical truth in the same indirect manner is valid within Proclus' philosophy. As Morrow argues, "[Proclus] and the Greeks were well aware of the utility of mathematics, and these applications to empirical problems demonstratable influenced the direction of mathematical imagination."70

There is some limitation to what more can be said about Proclus' understanding of the lower sciences as the two longest accounts of them are framed as recountings of the Pythagorean and Geminus' classifications; both of these schools of thought are challenged elsewhere, so neither can be known to be representative of Proclus' views. ${ }^{71}$ However, at their cores both present these lower sciences as the valid application of geometrical principles and arguments to imperfect sense objects, producing knowledge about their non-geometrical objects of study while relying on geometrical frameworks.

\section{Conclusion}

I have concluded that Proclus' geometric epistemology relies on the sense world in practice, even if the ontology exists free from physical things in its idea form. First, I established that Proclus

\footnotetext{
${ }^{69}$ Proclus, Commentary, 63-64.

${ }^{70}$ Morrow, "Introduction," lxii-iii.

${ }^{71}$ Proclus, Commentary, on Pythagoras 36-38 and on Geminus 38-42.
} 
grants mathematical objects a level of ontological fluidity; then I show that this fluidity is often taken advantage of by geometers to make up for shortcomings in human geometric ability by allowing them to leverage the physical world in their geometric study. In doing so, I resolve tension present in the text between the idealized mathematical schema and the mathematics actually performed by mathematicians and others. This conflict and resolution are uniquely accessible through Proclus' text because of its highly dialectical nature, as part philosophical study, part reader's supplement for Euclid: He asks and answers "how should we interact with mathematics" but also "how do we interact with it."

Through this, Proclus' commentary offers an insightful view predictive of modern mathematical philosophy and study. Guy Claessens shows that Kepler explicitly revives Proclus' epistemological structure in Harmonices Mundi (1619), while Mary Domski shows parallels between Proclus's and Newton's epistemologies in De Gravitatione (1687); in both cases, they highlight the use of the imagination to produce knowledge. ${ }^{72}$ I think that this comparison can be extended to modern mathematics, which has represented nearly two millennia of attempting to reach "pure and unalloyed understanding," the state of the god-geometer; while pen and paper and imaginary visualization still play an unquestionable role in mathematics, the starting points and movement between ontological realms are certainly more rigorously defined. Knowledge of the limitations and advantages of transformations between representations of a mathematical object is key for mathematical production and discovery today, offering a more rigorous examination of the ontological and epistemological questions studied by Proclus.

72 Claessens, "Kepler," 183-6; Mary Domski, "Newton and Proclus: Geometry, Imagination, and Knowing Space," The Southern Journal of Philosophy, Vol. 50, Issue 3 (Sept. 2012): 398-413. 


\section{References}

Brisson, Luc. "Chapter 18 of the De Communi Mathematica Scientia Translation and Commentary." In Iamblichus and the Foundations of Late Platonism, edited by Eugene Afonasin, John Dillon, and John F. Finamore. 37-50. Leiden: Brill, 2012.

Claessens, Guy. "Imagination as Self-knowledge: Kepler on Proclus' Commentary on the First Book of Euclid's Elements." Early Science and Medicine 16 (2011): 179-99.

Domski, Mary. "Newton and Proclus: Geometry, Imagination, and Knowing Space." The Southern Journal of Philosophy, Vol. 50, Issue 3 (Sept. 2012): 398-413.

Harari, Orna. "Proclus' Account of Explanatory Demonstrations in Mathematics and its Context." Archiv f. Gesch. d. Philosophie 90 (2008): 137-64.

MacIsaac, D. Gregory. "Geometrical First Principles in Proclus' Commentary on the First Book of Euclid's Elements." Phronesis 59, (2014): 44-98.

Manders, Kenneth. "The Euclidean Diagram (1995).” In The Philosophy of Mathematical Practice, ed. Paolo Mancosu, 80-133. Oxford University Press, 2008.

Morrow, Glenn R. "Introduction.” In A Commentary on the First Book of Euclid's Elements.

Nikulin, Dmitri. "Imagination and Mathematics in Proclus." Ancient Philosophy 28 (2008): 153-172.

Plato. The Republic. Translated by Benjamin Jowett. The Internet Classics Archive, Massachusetts Institute of Technology.

Proclus Lycius. A Commentary on the First Book of Euclid's Elements. Translated by Glenn R. Morrow. Princeton: Princeton University Press, 1992.

Rabouin, David. "Proclus' Conception of Geometric Space and Its Actuality." In Mathematizing Space, ed. Vincenzo De Risi, 105-42. Switzerland: Springer, 2015.

Raymond, Dwayne. "From a Particular Diagram to a Universal Result: Euclid's Elements, Book 1." Apeiron, vol. 44 (2001): 211-218.

Sheppard, Anne. "Phantasia and Mathematical Projections in Iamblichus." Syllecta Classica, Vol. 8 (1997): 113-120. 